\title{
Reply to Frane: Epidermolysis Bullosa Pruriginosa: A Systematic Review Exploring Genotype-Phenotype Correlation
}

\author{
Whan B. Kim ${ }^{1}$ - Afsaneh Alavi ${ }^{2,3} \cdot$ Scott Walsh ${ }^{2,4} \cdot$ Elena Pope $^{2,5}$
}

Published online: 18 July 2015

(c) Springer International Publishing Switzerland 2015

With regard to our recent article, Frane [1] explains that the justifications given for not adjusting for multiple comparisons are false. In reviewing the statistical literature, the authors agree that the criteria provided in the article are not based on robust statistical principles but rather stem from popular statistical heuristics [2], and such justifications should not be perpetuated throughout the literature.

However, the irrelevance of the justifications should not detract from the message of our paper. The systematic review seeks to explore genotype-phenotype correlations in epidermolysis bullosa pruriginosa (EBP) in light of existing discrepancies reported by individual case reports or case series.To this end, the article has identified all existing cases of mutation-verified EBP in the literature. In doing so, the review serves as a summary of the literature available for EBP, as illustrated in Table 2 of the article [3]. Although, perhaps, less emphasis should be put on interpreting the significance of the statistical hypothesis tests, the collation of patient genotype and phenotype data with summary statistics, confidence intervals and unadjusted $p$ values

Elena Pope

elena.pope@ sickkids.ca

Whan B. Kim

whan.kim@medportal.ca

1 Michael G. DeGroote School of Medicine, McMaster University, 1280 Main St W, Hamilton, ON L8S 4L8, Canada

2 Division of Dermatology, Department of Medicine, University of Toronto, Toronto, ON, Canada

3 Women's College Hospital, Toronto, ON, Canada

4 Sunnybrook Health Sciences Centre, Toronto, ON, Canada

5 University of Toronto Hospital for Sick Children, Toronto, ON, Canada reported can serve to direct future study designs, and to potentially provide critical information with respect to diagnosis, prognostication, and patient care.

It is also worth noting that for a rare genetic disease such as EBP it is often very difficult, if not impossible, to obtain enough cases to sufficiently power a study that addresses genotype-phenotype comparisons for multiple relevant endpoints, while adjusting for multiple comparisons. Although it is imperative to adhere to sound statistical principles, it is critical that emphasis on statistical testing not preclude or detract from the efforts made to identify, summarize, and translate available data for the benefit of clinicians, patients, and family members.

Acknowledgments The authors would like to acknowledge Dr. Lisa Strug, PhD, ScM, Dalla Lana School of Public Health, University of Toronto, Toronto, ON, Canada, for her contributions to the statistical content of this work.

\section{Compliance with ethical standards}

Funding No funding was received for the preparation of this letter.

Conflicts of interest Whan Kim, Afsaneh Alavi, Scott Walsh, and Elena Pope report no conflicts of interest.

\section{References}

1. Frane A. On false justifications for disregarding multiplicity. Am J Clin Dermatol. doi:10.1007/s40257-015-0139-3.

2. Armstrong RA. When to use the Bonferroni correction. Ophthalmic Physiol Opt. 2014;34:502-8.

3. Kim W, Alavi A, Walsh S, Kim S, Pope E. Epidermolysis bullosa pruriginosa: a systematic review exploring genotype-phenotype correlation. Am J Clin Dermatol. 2015;16:81-7. 